\title{
LATE LIVER METASTASIS 20 YEARS AFTER THE INITIAL DIAGNOSIS: A CASE REPORT OF METACHRONOUS HORMONE DEPENDENT TUMORS OF BREAST AND ENDOMETRIUM
}

\author{
Ivan Ilić, Maja Jovičić Milentijević, Aleksandar Milićević, Milica Stanković, Aleksandra Radičević \\ Favulty of Medicine, University of Niš, Niš, Serbia
}

\begin{abstract}
Breast cancer is the most common cancer and a significant cause of morbidity and mortality in the female population worldwide. The liver is the third most common metastatic site for invasive breast malignancy besides bones, lungs, and brain. Breast cancer has been linked with metachronous bone, endometrial, colon/rectal, connective tissue (sarcoma), leukemia, lung, ovary, or thyroid cancer. Studies have shown an increased risk of secondary malignancies in women treated for breast malignancy in connection to adjuvant treatment in certain cases.

We present a case of a 71 -year -old woman who was diagnosed with breast cancer 20 years ago. The primary diagnosis was invasive lobular breast cancer localized in the left lower lateral quadrant. Micromorphological, histochemical and immunohistochemical analyses rendered diagnosis inconclusive due to lack of tissue so after 4 months rebiopsy was performed. Clinico-pathological correlation of the second biopsy was in favor of liver metastasis of partially hormonedependent breast cancer. Immunohistochemistry was vital for the diagnosis of the liver biopsy, in particular GATA3 positivity and vimentin negative staining which helped us exclude endometrial cancer metastasis which was diagnosed before the initial liver biopsy. GATA 3(+)/vimentin(-) panel proved to be superior to GCDFP-15 and mammaglobin in proving the breast origin of the secondary tumor deposit.

Liver metastasis from primary breast cancer can in certain cases occur many years after the initial diagnosis which shows the importance and necessity for long- term follow-up of these patients, while considering the possibility of metachronous tumors as well.
\end{abstract}

Key words: breast cancer, liver metastases, immunohistochemistry, metachronous tumors.

\section{Introduction}

Breast cancer is the most common cancer and a significant cause of morbidity and mortality in the female population worldwide. In around $20 \%-30 \%$ of cases distant metastases occur [1].

Breast cancer usually disseminates to bones, lungs, liver, and brain by hematogenous spread; the liver is the third most common metastatic site for invasive breast malignancy [2].

The status of estrogen receptor (ER), progesterone receptor (PR), and human epidermal growth factor receptor 2 (HER-2) in primary and metastatic breast cancers was evaluated and links between ER, PR, and HER-2 with various localisations of breast cancer (including liver) were investigated. ER+ or PR+/HER-2- (luminal A) subtypes were present in $75.0 \%$ of the cases [3].

A panel of immunohistochemical markers necessary for confirming the breast origin of the metastatic deposit consists of: GATA3, mammaglobin and gross cystic disease fluid protein 15 (GCDFP-15). In clinical practice, GATA3 immunohistochemistry stains a greater number

Correspondence to: Ivan Ilić M.D., Ph.D

Faculty of Medicine, Dr. Zoran Djindjić Blvd., 81, 18000 Niš, Serbia Phone: +381637268033

E-mail: ilicko81@gmail.com

Received November $10^{\text {th }}, 2020 /$ Accepted January $23^{\text {rd }}, 2021$ of primary and secondary breast carcinomas than both mammaglobin and GCDFP-15 [4].

If the tumor reoccurs five or more years after the initial diagnosis, it would indicate a long-dormant period of undetectable metastases. Recent studies have focused on predicting the risk of late breast cancer recurrence by investigating clinical factors, subtypes, genes, and immune status. In a recent study early to mid recurrence was associated with more aggressive clinical progression and less favourable parameters (greater tumor diameter, more frequent lymph node metastases, higher tumor grades, later stages, and negative ER and PR hormone status) compared to survivors and the patients with late breast cancer recurrence [5].

Metastasising breast carcinomas need to be differentiated from synchronous/metachronous gynecological malignant tumors with an estrogen receptor/cytokeratin 7-positive (ER+/CK7+) and mammaglobin-positive immunohistochemical profile, which can be achieved by using GATA3 immunohistohemical stain which proved to be highly useful in diagnosing breast carcinomas, both primary and metastatic [6].

Here we report a case of a patient with breast cancer liver metastasis 20 years after primary diagnosis, which was confirmed on a liver biopsy by pathohistological and immunohistochemical analysis. 


\section{Materials and Methods}

Liver biopsy was formalin- fixed (10\% neutral buffered formalin), processed, paraffin- embedded, and then stained using standard hematoxylin and eosin(H\&E) stain. The specimen was also stained histochemically using alcian blue- periodic acid-Schiff (AB-PAS) staining method at $\mathrm{pH} 2.5$.

The tissue from the paraffin moulds was cut into $4 \mu \mathrm{m}$ thick sections and placed on aptaca slides. In brief, $4 \mu \mathrm{m}$ thick tissue sections were deparaffinized in xylene and rehydrated in a graded series of ethanol, and deionized water. Following adequate heat-induced antigen retrieval procedure, the endogenous peroxidase activity was quenched, and the slides were rinsed thoroughly with phosphate- buffered saline. The primary antibodies (Cytokeratin mouse clone AE1/AE3, mouse monoclonal CA125-clone M11, rabbit HER2-polyclonal, mouse monoclonal Estrogen receptor-clone 1D5, mouse monoclonal Progesteron receptor-clone PgR 636, and mouse monoclonal vimentin-clone V9 were applied and the slides were incubated in a water bath for one hour at room temperature. Appropriate positive and negative controls were included in every immunostaining procedure. For the visualization reaction, a standard immunoperoxidase detection system was applied according to the manufacturer's instructions (DAKO LSAB2R system-HRP, Dako, Denmark), and diaminobenzidine was used as a chromogen. Slides were afterward counterstained with Mayer's hematoxylin, dehydrated, and mounted.

Using autostainer Ventana Benchmark GX tissue sections were stained with monoclonal ready to use antibodies GATA3-mouse clone L50-823, GCDFP-15 (EP1582Y) Rabbit Monoclonal Antibody, and p16CINtec.

\section{Case Report}

We present a case of a 71- year- old woman who was diagnosed with breast cancer 20 years ago. The primary diagnosis was invasive lobular breast cancer localized in the left lower lateral quadrant. Soon after primary diagnosis left mastectomy was performed, and the patient received radiation therapy afterwards. During a detailed follow-up 3 years ago she was diagnosed with endometrial cancer and treated accordingly. During the time of the liver biopsy on scintigraphy, multiple tumor deposits were noted.

Initially, the pathohistological examination of a liver biopsy sample showed the focal presence of dense infiltrates of inflammatory and reactive cells predominantly with ovoid and irregular morphology, abundant and occasionally eosinophile granular cytoplasm resembling histiocytes. Histochemical and immunohistochemical staining of these cells showed positivity for toluidine blue while it was negative on AB PAS, CK7, S100, CD 68, and mammaglobin. Additional immunohistochemical analyses weren't possible due to lack of tissue, so a rebiopsy was suggested.
After 4 months, the rebiopsy was performed which showed a marginal area of proliferative connective tissue with individual and small groups of discohesive cells (Figure 1.A, 1.B). The nuclei of these cells were localized marginally and mucins were not observed in the cytoplasm of individual cells (Figure 1.C). Immunohistochemical staining showed CKAE1/E3 (Figure 1.E), GATA3 (Figure 2.A), ER positivity and Ca125 (Figure 2.B, 2.E), GCDFP-15 (Figure 1.D), p16 (Figure 2.F), PR (Figure 2.C), HER2 (Figure 2.D), vimentin negative staining (Figure 1.F).

Immunohistochemistry, micromorphology and clinicopathological correlation were in favor of the liver metastasis of partially hormone dependent breast cancer (strong nuclear expression of estrogen receptors on most tumor cells and negative expression of progesteron receptors as well as HER 2 receptor negative staining).

\section{Discussion}

Breast cancer most frequently metastases to the lymph nodes, liver, bones, and lungs. Liver metastases from breast cancer may present asymptomatically or with digestive tract system symptoms like bloating, ascites, palpable abdominal mass, jaundice, or weight loss [1].

Breast cancer has been linked with metachronous bone, endometrial, colon/rectal, connective tissue (sarcoma), leukemia, lung, ovary, or thyroid cancer. The most common second primary cancer in breast cancer patients was that of the opposite breast $(23.9 \%)$, it was detected on an average of 7 years after the first cancer was detected. The risk of endometrial, thyroid, and ovarian cancer was higher than that of the general population [7].

Studies have shown an increased risk of secondary malignancies in women treated for breast malignancy in connection to adjuvant treatment in certain cases. Higher endometrial cancer incidence has been observed in adjuvant trials of tamoxifen [8]. Endometrial cancer was not entirely caused by tamoxifen but also influenced by parameters like reproductive and genetic factors, obesity, and in some cases unknown factors that could lead to estrogen excess [9]. Women in the postmenopausal period with symptoms that arouse suspicion for endometrial hyperplasia or cancer and who are using tamoxifen should be evaluated in terms of therapy and diagnostics [10].

A majority of breast cancers (more than $90 \%$ ) are diagnosed as no special type (NST) invasive carcinoma, which is characterized by tubule formation that resembles adenocarcinoma, the main differential diagnosis of metastatic breast carcinoma in the liver is primary intrahepatic cholangiocarcinoma. Histologically, breast carcinoma consists of atypical epithelial cells with lightly monomorphic or highly pleomorphic nuclei arranged in tubular, glandular, cribriform, or solid patterns [11]. In our case tumor cells had a discohesive pattern resembling lobular carcinoma unlike endometrial adenocarcinoma which has a glandular pattern. 


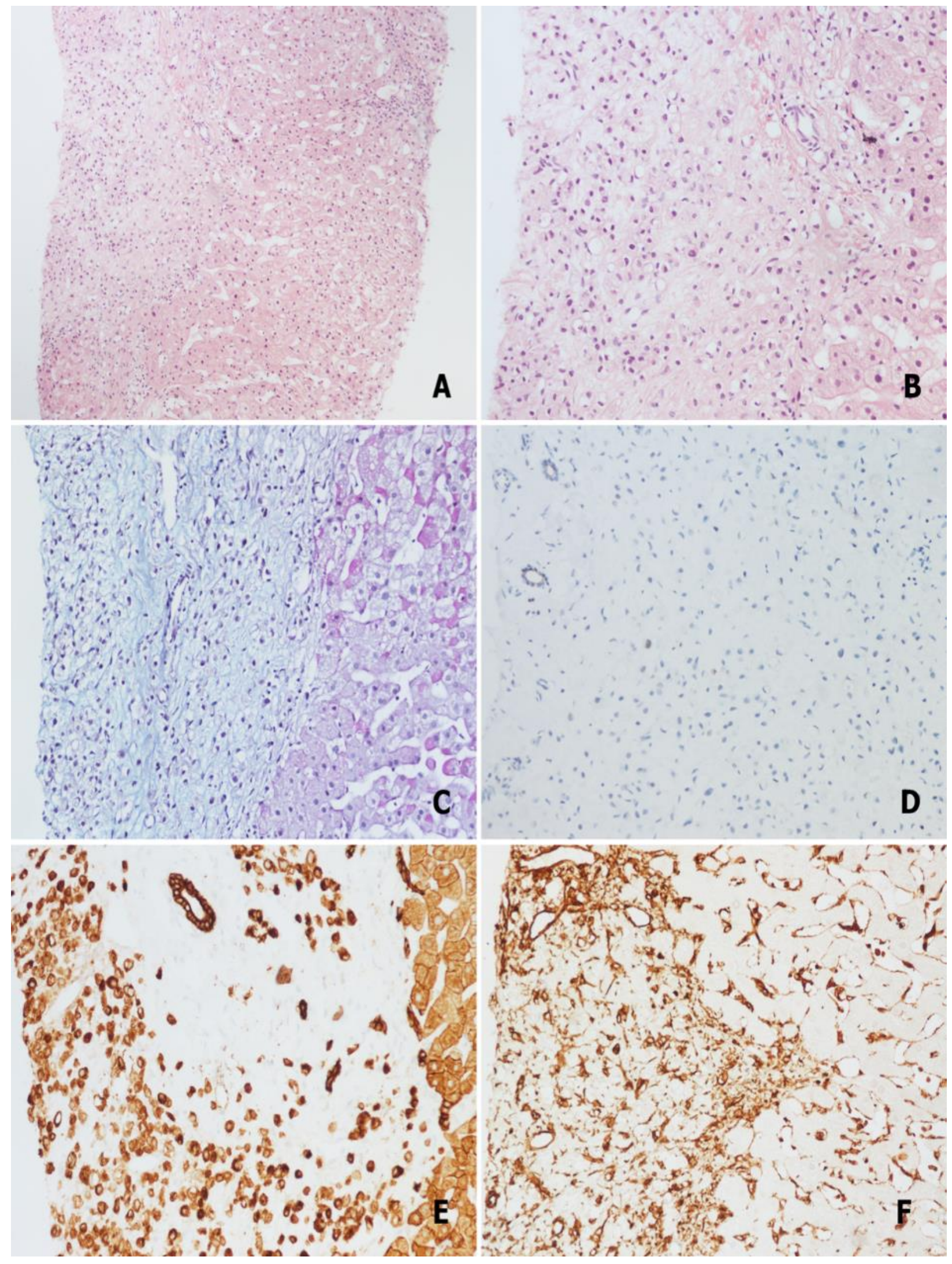

Fig. 1 A. Well demarcated metastatic focus on liver needle biopsy (HE x100); B. Higher magnification shows a relatively uniform population of cells some with "signet ring" features (HE x200); C. AB PAS histochemical stain showed cells without the mucin component (AB PAS x200); D. GCDFP-15 negative stain for apocrine differentiation (LSAB x200); E. CKAE1/AE3 showed strong, diffuse positivity (LSAB x200); F. Vimentin stain was negative in tumor cells (LSAB x200). 


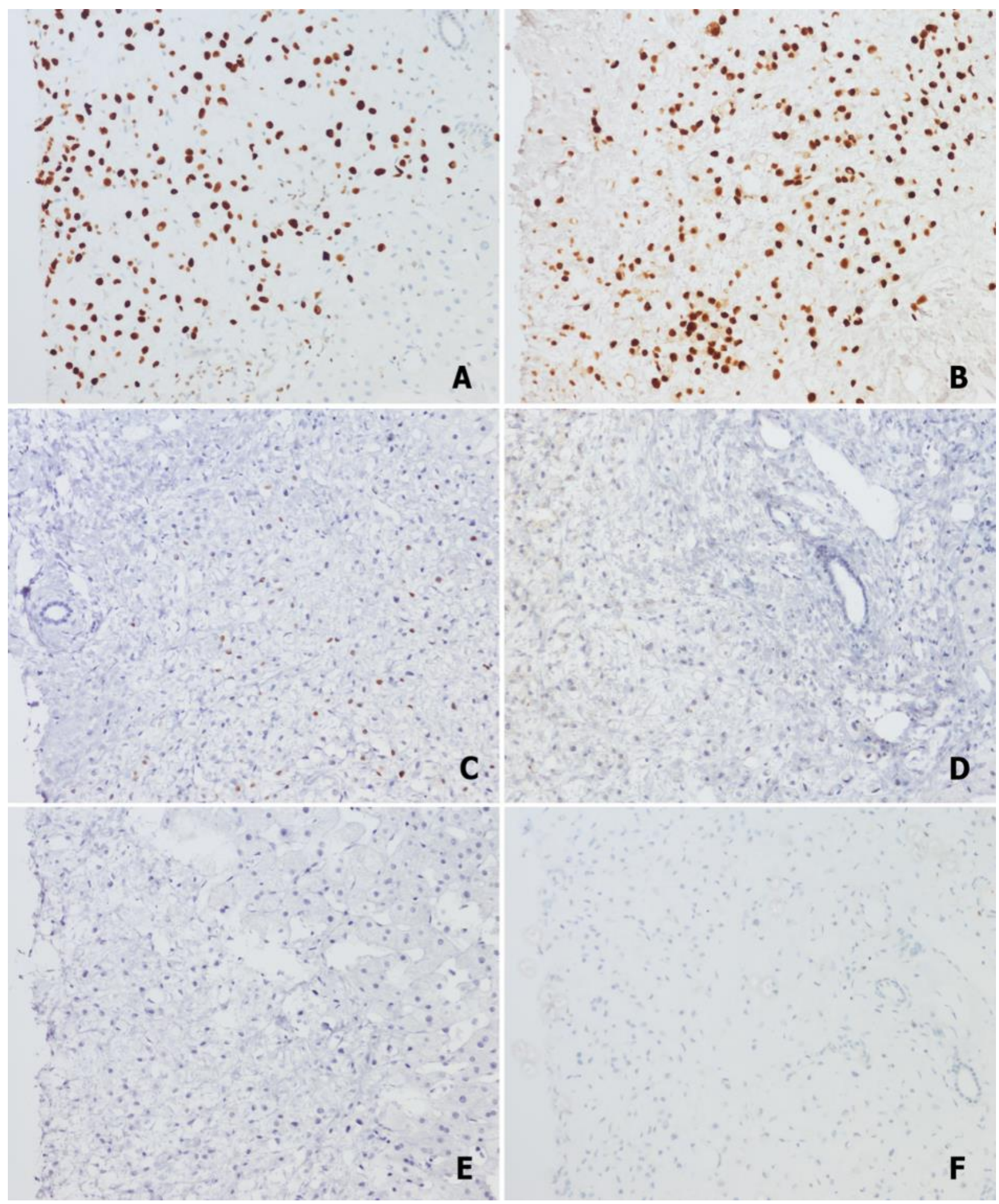

Fig. 2 A. GATA3 stain showed strong nuclear positivity in tumor cells (LSAB x 200); B. Strong nuclear positivity of ER receptors in all tumor cells (LSAB x200); C. Weak nuclear PR receptor positivity in minority of tumor cells (LSAB x200); D. Weak membranous stain of HER2 in minority of tumor cells (LSAB x200); E. Negative immunoreactivity for CA125 stain (LSAB x200); F. Negative immunoreactivity for p16 stain (LSAB x200). 
Invasive lobular carcinoma is the second most common histologic type of breast carcinoma after ductal invasive carcinoma (NST type in the majority of cases). Morphologically, in most cases, this histologic type is characterized by infiltration of single-cell file type or discohesive epithelial cells, which consist of monotonous nuclei and inconspicuous nucleoli [11]. In the case of metastasing breast cancer confirmation of metastatic disease and identification of its primary origin is further complicated by the existence of multiple (two or more) primary malignancies, such as synchronous/ metachronous breast cancer and gynecological malignant tumors with an estrogen receptor-positive/cytokeratin 7-positive $(\mathrm{ER}+/ \mathrm{CK} 7+)$ and mammaglobin-positive immunohistochemical profile. GATA3 expression has been reported as a useful marker for differential diagnosis between malignancies from other localisations and breast carcinomas (both primary and metastatic) [6].

A comparison of hormone receptors and HER2 receptor immunohistochemical expression between primary and metastatic breast carcinoma in the liver was also useful in establishing the differential diagnosis. In addition, immunohistochemical studies for tissue-specific markers, such as gross cystic disease fluid protein 15 (GCDFP-15) and mammaglobin, will be helpful [11]. Considering the aforementioned differential diagnoses immunohistochemical panel that showed CKAE1/E3, GATA3, ER positivity and CA125, GCDFP-15, p16, PR, HER2, and vimentin negativity was used. Our data (ER+, PR-,HER-2-) matches the result of the study [3] in terms that ER+ or PR+/HER-2- (luminal A) subtypes were predominant in the sites of liver metastases $(75.0 \%)$.

\section{References}

1. Yazdani A. Risk factor of liver metastases in breast cancer. Arch Cancer Sci Ther 2019; 3:63-65.

2. Hess KR, Varadhachary GR, Taylor SH, Wei W, Raber MN, Lenzi R, et al. Metastatic patterns in adenocarcinoma. Cancer 2006; 106:1624-1633.

3. Koo JS, Jung W, Jeong J. Metastatic breast cancer shows different immunohistochemical phenotype according to metastatic site. Tumori 2010; 96: 424-432.

4. Sangoi AR, Shrestha B, Yang G, Mego O, Beck AH. The novel marker GATA3 is significantly more sensitive than traditional markers mammaglobin and GCDFP15 for identifying breast cancer in surgical and cytology specimens of metastatic and matched primary tumors. Appl Immunohistochem Mol Morphol 2016; 24:229-237.

5. Takeshita T, Yan Li, Asaoka M, Rashid O, Takabe K. Late recurrence of breast cancer is associated with pro-cancerous immune microenviroment in the primary tumor. Sci Rep 2019; 15:16942.
In our case, tumor cells were partially hormone- sensitive and HER-2 negative.

Other factors besides the hormonal (tamoxifen therapy and other causes of hypestrogenism) might be at play, such as: HER2- negative status, age, body weight, genetic predisposition, etc.

\section{Conclusion}

This case report highlights a late manifestation of liver metastasis from breast cancer, 20 years after the primary diagnosis. Considering that our patient had a previous history of breast malignancy, clinical suspicion for metastatic disease was justified. Also, clinical and radiological features may be non-specific, sometimes misleading, so the definitive diagnosis represents a challenge for the pathologist.

Late metastasis may be attributed to its hormonal profile which also resulted in the formation of metachronous endometrial cancer a year before the liver metastasis. Immunohistochemistry was vital for the diagnosis on the liver biopsy, in particular GATA3 positivity and vimentin negative staining, which helped us exclude endometrial cancer metastasis. GATA 3(+)/vimentin(-)panel proved to be superior to GCDFP-15 and mammaglobin in proving the breast origin of the secondary tumor deposit.

Generally, liver metastasis from breast cancer may occur many years after the primary diagnosis, which shows the importance and necessity for long -term follow-up of these patients.

6. De Lara S, Parris TZ, Ronnerman EW, Helou K, Kovacs A. GATA3 as a putative marker of breast cancer metastasis - a retrospective immunohistochemical study. Breast J 2018; 24: 184-188.

7. Schenker JG, Levinsky R, Ohel G. Multiple primary malignant neoplasms in breast cancer patients in Israel. Cancer 1984; 54:145-150.

8. Kirova YM, Rycke De, Gambotti L, Pierga JY, Asselain B, Fourquet A. Second malignancies after breast cancer: the impact of different treatment modalities. Br J Cancer 2008; 98:870-874.

9. Mellemkjaer L, Friis S, Olsen JH, Scelo G, Hemminki K, Tracey E, et al. Risk of second cancer among women with breast cancer. Int J Cancer 2006; 118:2285-2292.

10. Kim JY, Song HS. Metachronous double primary cancer after treatment of breast cancer. Cancer Res Treat 2015; 47:64-71.

11. Park JH, Kim JH. Pathologic differential diagnosis of metastatic carcinoma in the liver. Clin Mol Hepatol 2019; 25:12-20. 